\title{
A Relative Frame Representation for Fixed-Time Bundle Adjustment in SFM
}

\author{
Steven Holmes, Gabe Sibley, Georg Klein and David W Murray
}

\begin{abstract}
A successful approach in the recovery of videorate structure from motion is to allow the camera to keep track of its position in every frame assuming the recovered set of scene landmarks is fixed in $3 \mathrm{D}$, and then to use the poses in a subset of separated frames, or keyframes, to initialise further landmark structure. The landmark structure and keyframe poses are optimised in a bundle adjustment. Unfortunately this monolithic bundle adjustment has cubic complexity.

This paper shows how representing landmarks and camera poses in relative frames, and by temporarily removing certain measurements, introduces a conditional indepedence which allows the bundle adjustment to be split into two parts. One "local" part involves the most recent keyframes and associated landmarks, and runs in constant time. The other "global" part deals with older keyframes and structure, and runs, as ever, in cubic time. Three important outcomes are: (i) the fixedtime local adjustment allows exploratory map-building to keep pace with camera pose tracking; (ii) it produces statistically consistent results; and (iii) referencing to relative frames means that any update in positions from the global adjustment are immediately incorporated in the local fixed-time adjustment.

The relative frame approach is applied to the parallel tracking and mapping method for structure from motion, and its results shown to be identical, and the exploratory map building phase shown to maintain fixed time performance.
\end{abstract}

\section{INTRODUCTION}

Real-time visual structure from motion for large areas is plagued by two distinct problems. The first is that the complexity of finding an optimum and global solution, one that involves all measurements and parameters, scales poorly with the number of landmarks mapped. The second is that algorithms which attempt to finesse the problem of complexity - say by neglecting parameters or by neglecting small dependencies in the problem - are often overconfident, placing too small error estimates on their suboptimal solutions.

Amongst algorithms which seek a global solution the lowest complexity is achieved by FastSLAM [1], in which a factorization exploits the landmark estimations' independence, conditioned on the trajectory of the sensor. Its complexity is is $O(P \log L)$ in the number of landmarks $L$, achieved by using a particle-filter with $P$ particles to represent the trajectory, and a set of Extended Kalman Filters, each conditioned on one of the particles, to estimate the landmark positions. FastSLAM is consistent only for limited areas [2], beyond which the number of particles required to represent the pdf adequately becomes prohibitive.

The authors are with the Department of Engineering Science, University of Oxford, Oxford OX1 3PJ, UK. www.robots.ox.ac.uk/ActiveVision [sah,gsibley,gk,dwm]@ robots.ox.ac.uk

This work was supported by Grants GR/S97774 and EP/D037077 from the UK Engineering and Physical Science Research Council. SAH is supported by an EPRSC Research Studentship.
Moving up in complexity are Kalman-based solutions [3] [4] [5] which exploit the static nature of the landmarks to reduce the complexity of the general Extended Kalman Filter (EKF) from $O\left(L^{3}\right)$ to $O\left(L^{2}\right)$. These filters always produce inconsistent estimates [6], [7], [8] due to the shortcomings of linearization about an incorrect operating point. However the real sticking point for these filters is that their quadratic complexity puts a tight constraint on the number of landmarks which can be mapped.

Another step up in complexity brings one to full optimizations, all variants of Bundle Adjustment [9]. Of particular note in this paper is Parallel Tracking and Mapping (PTAM) [10] which achieves video-rate recovery of structure from motion involving several thousand landmarks by separating the problem of mapping from that of tracking the camera pose from frame to frame. However the method is still limited by cubic complexity in the mapping phase. The $O\left(K^{3}\right)$ complexity is in the number of camera poses (or keyframes) $K$ rather than the number of landmarks, in which the complexity is only linear. Other algorithms mitigate the effects of cubic complexity either by optimising only a subset of the parameters that we are interested in [11], or by pausing only infrequently to calculate the full optimization [12].

One approach to reducing complexity is to use a submapping strategy [13], [14], [15], [16]. In [16] the approach is to marginalize out the previous map when a new map is started. The full solution is found by back-projecting information to the old maps. The other approaches use an EKF for the submaps and then optimize the transformations between them. These schemes can be adapted to continuous exploration by continually adding new submaps, but to create a global map still needs an optimization with cubic complexity. Eade and Drummond's real time monocular SLAM system [14] based on the Atlas framework [13], for example, splits the map into a set of submaps, called nodes, in which the observation model is nearly linear. In each submap the landmarks are represented in the node's coordinate frame and are updated using an information filter. Adjacent nodes have common features which are used to calculate the transformation between the nodes. These transformations are then optimized subject to the constraints that loops of transformations should be identities.

It appears then that approaches which optimize using all the measurements always need a step with cubic complexity to produce consistent results. Moreover, in these approaches mapping cannot continue until the optimization is finished. In contrast, all approaches which use all the measurements and which have less than cubic complexity eventually produce inconsistent results. 
Relative bundle adjustment [17] builds a map of transformations between camera poses and landmark positions. The global position of a camera is not constrained to be the same when multiple paths of transformations give its position. The optimal solution for this representation can be estimated in constant time but projecting the solution to a single global Euclidean frame is still a cubic operation.

Accepting that the optimal map using all of the measurements incurs a cubic cost, our aim in this paper is to develop an approach which carefully subsamples the available measurements to allow exploration to proceed in constant time, while producing a conservative estimate of the map - one that is not over-confident. We also explain how the approach can be extended so that the globally optimal estimate can be obtained without hindering the performance of the constant time exploration.

The approach is based on a combination of two methods introduced recently by the authors - the relative representation for bundle adjustment [17], and the parallel tracking and mapping framework for monocular structure from motion [10]. In the relative representation of bundle adjustment the location of each landmark is represented in the camera frame from which it was first observed and the camera frames themselves are represented relative to previous camera frames. This allows the local mapping in the vicinity of the camera to be carried out in constant time, while the global optimization can be carried out on the previously mapped areas. If the global optimization on the previously mapped area makes a correction to the more recent and current camera poses, the correction is immediately and automatically taken in account because the more recent camera poses are referred to the previous ones. Another benefit is that loop closing can be carried out as a separate process from the local mapping.

We contend that this combined approach possesses many of the most important characteristics for a visual structure from motion system engaged in active real-time scene exploration, viz:

1) constant time exploration,

2) linear storage requirements,

3) constant time topological mapping, and

4) provision of a globally optimum, and de facto consistent, map.

This paper is concerned principally with the first three of these. As noted earlier, the fourth is a solved problem with irreducible complexity - though providing sound data and good initial parameters for it is always of interest.

The paper continues by describing relative frame bundle adjustment, and Section III describes the integration of the relative representation with parallel tracking and mapping. Experimental results are presented in Section IV which show the equivalence of numerical estimates produced by the standard and relative frame approaches, and which demonstrate the ability to explore in constant time. Conclusions are drawn and future work outlined in Section V.

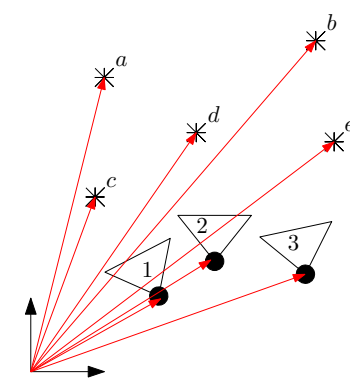

(a)

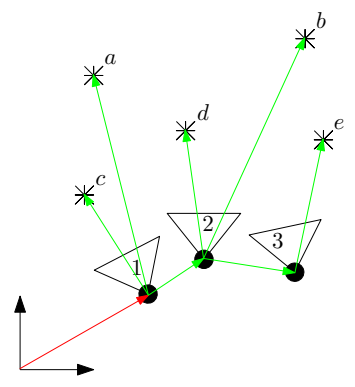

(b)
Fig. 1. (a) In the global representation all the keyframes and landmarks are represented in the global coordinate frame. (b) The relative representation has only the first keyframe represented in the global coordinate frame. all other landmark positions and keyframe poses are represented relative to other keyframes.

\section{Relative Frame Bundle Adjustment}

Bundle adjustment [9] [18] seeks to find the optimum set of scene parameters $\boldsymbol{\xi}^{*}$ by minimising a cost function expressing the sum of squared differences between the estimated image locations $\mathbf{f}(\boldsymbol{\xi})$ and the measured image locations $\mathbf{Z}$,

$$
\boldsymbol{\xi}^{*}=\arg \min _{\boldsymbol{\xi}}(\mathbf{Z}-\mathbf{f}(\boldsymbol{\xi}))^{\top}(\mathbf{Z}-\mathbf{f}(\boldsymbol{\xi})) .
$$

The standard way to solve this minimization is using a partitioned Levenberg-Marquardt algorithm [18]. Unlike algebraic methods, the minimization occurs in measurement space, and the underlying parameterization is directly in terms of physical entities, specifically the set of camera poses in keyframes and the set of landmark locations in three dimensions. An important aspect of bundle adjustment (including relative frame bundle adjustment) is that the measurement corresponding to a landmark is independent of all other landmarks, allowing a partitioned LM-algorithm to run with complexity $O(L)$ in the number of landmarks $L$.

In standard bundle adjustment all the parameters (landmarks and camera keyframe poses) are referred to a single global coordinate frame which may be chosen arbitrarily. By contrast, in relative frame bundle adjustment, each landmark is referred to the camera keyframe in which it was first instantiated. Keyframes themselves are referenced to the coordinate system of a previous keyframe - the particular keyframe chosen is that which has the largest number of measurements from landmarks in common.

Figures 1(a) and (b) shows a simple example using the standard and relative frame approaches, respectively. Three keyframes and five landmarks are shown. Landmarks $a$ and $c$ are first detected in keyframe $1, b$ and $d$ in keyframe 2 , and landmark $e$ in keyframe 3 . In the relative frame representation homogeneous Euclidean transformations, $\mathrm{E}_{i j}$, explicitly link frames in the obvious way, so that for example that between frame 3 and the global frame (here indexed with $0)$ is $\mathrm{E}_{03}=\mathrm{E}_{01} \mathrm{E}_{12} \mathrm{E}_{23}$. Any $\mathrm{E}_{i j}=\mathrm{E}_{j i}{ }^{-1}$, of course.

A measurement $\mathbf{Z}_{i k}=(u, v)^{\top}$ in the $k^{\text {th }}$ keyframe of the $i^{\text {th }}$ landmark, that is itself referred to the the $j^{\text {th }}$ keyframe, is assumed to arise from perspective projection into the image plane

$$
\left[\begin{array}{c}
\mathbf{Z}_{i k} \\
1
\end{array}\right] \sim \mathrm{C}[\mathrm{I} \mid \mathbf{0}] \mathrm{E}_{k j}\left[\begin{array}{c}
\mathbf{X}_{j}^{i} \\
1
\end{array}\right]
$$




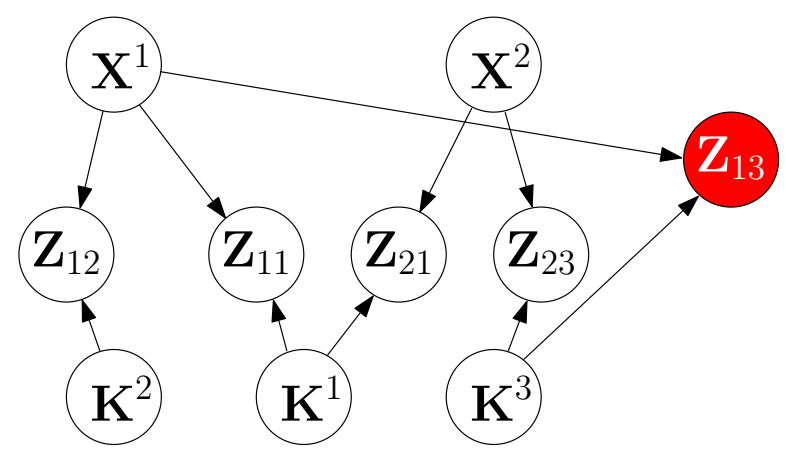

Fig. 2. The Bayes net for a simple bundle adjustment containing 3 keyframes, one set of landmarks observered by keyframes 1 and 3 and a set of landmarks observed by all the keyframes.

where $\mathrm{C}$ is the intrinsic camera calibration, which is assumed known. (In practice, a correction for radial distortion is also applied.) Returning to the example in Figure 1(b), an observation of landmark $a$ in keyframe 3 would be given by

$$
\left[\begin{array}{c}
\mathbf{Z}_{a 3} \\
1
\end{array}\right] \sim \mathrm{C}[\mathrm{I} \mid \mathbf{0}] \mathrm{E}_{32} \mathrm{E}_{21}\left[\begin{array}{c}
\mathbf{X}_{1}^{a} \\
1
\end{array}\right] \text {. }
$$

When a landmark is being observed in the keyframe to which it is referred, the $\mathrm{E}_{k j}$ drops out as the identity matrix.

Apart from the changes in referencing, the parameter set in the relative frame representation is of similar form to that in standard bundle adjustment

$$
\boldsymbol{\xi}=\left[\mathbf{K}^{1}, \ldots, \mathbf{K}^{K}, \mathbf{X}_{k}^{1}, \ldots, \mathbf{X}_{k}^{L}\right] .
$$

At each step of the LM optimization, the Jacobian, J, of derivates of the measurements $\mathbf{Z}$ with respect to the parameter set $\mathbf{X}$ has to be computed. Here there is a substantive difference. Whereas in standard bundle adjustment there are always just two components in the Jacobian for each measurement (the derivatives with respect to (i) the landmark's 3D position $\mathbf{X}$ and (ii) the pose $\mathbf{K}$ in the global frame of the keyframe associated with the measurement), it is obvious enough that in the relative frame representation the Jacobian will be more densely populated. There is again one component associated with the underlying landmark, but now the 3D position is referred to a particular keyframe. The derivative of the measurement in the $k^{\text {th }}$ keyframe of the $i^{\text {th }}$ landmark (which is referred to the $j^{\text {th }}$ keyframe) is

$$
\frac{\partial \mathbf{Z}_{i k}}{\partial \mathbf{X}_{j}^{i}}
$$

Instead of the single component for the keyframe, the Jacobian in relative frame bundle adjustment has as many components as there are links in the chain from landmark keyframe to observation keyframe, for example ${ }^{1}$

$$
\frac{\partial \mathbf{Z}_{i k}}{\partial \mathbf{K}^{i+1}}, \frac{\partial \mathbf{Z}_{i k}}{\partial \mathbf{K}^{i+2}}, \ldots \frac{\partial \mathbf{Z}_{i k}}{\partial \mathbf{K}^{k}} .
$$

In the case that the landmark is specified in the keyframe in which it is observed, then no derivative with respect to a keyframe is needed.

Even though the Jacobian in the relative frame representation is denser than that in standard bundle adjustment, it is still sparse in absolute terms because a landmark tends to be viewed from a subset of the keyframes which are near to

\footnotetext{
${ }^{1}$ In Eq. (3) the indices count up "nicely", following Figure 1. In general of course this will not be the case.
}
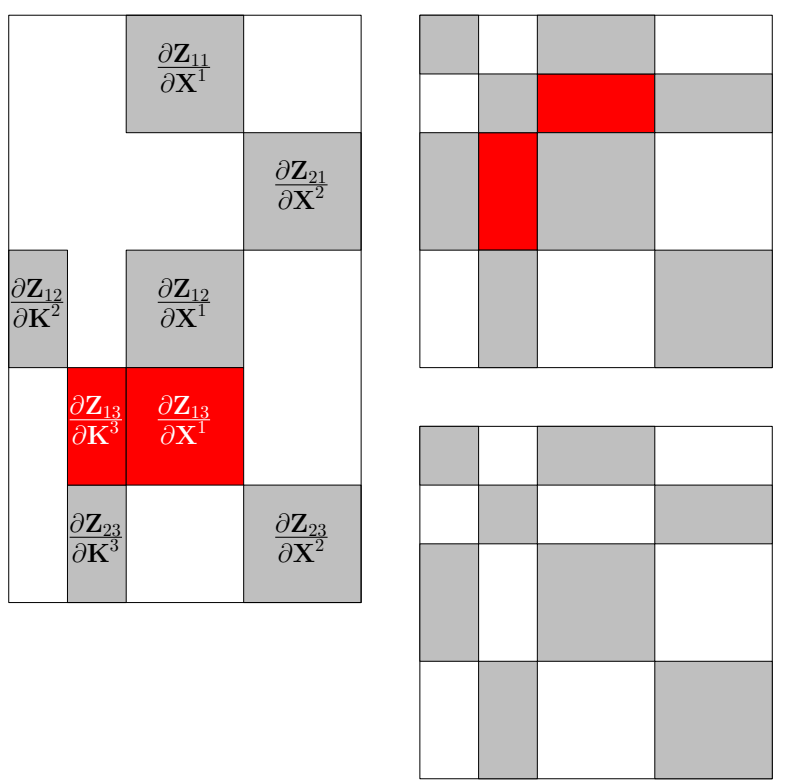

Fig. 3. The observation Jacobian, left, and the information matrix, top right, for the Bayes net shown in figure 2. Keyframe 1 is assumed to be fixed so there are no derivatives with respect to it. The red (dark) rows in the Jacobian and the red blocks in the information matrix are the components which correspond to the measurements $\mathbf{Z}_{13}$. The infomation matrix in the bottom right is for the reduced system in which the measurements $\mathbf{Z}_{13}$ have been discarded, this is a conservative estimate for upper information matrix.

each other. The minor disadvantage in computational cost is outweighed by the information processing advantages which are explained now.

\section{A. Conservative estimates and conditional independence}

The information matrix, $\mathcal{I}$, for the converged system is calculated from the measurement Jacobian as $\mathcal{I}=\mathrm{J}^{\top} \mathrm{J}$. Provided the data association is correct, each additional measurement adds rows to the Jacobian and hence adds information to the estimate. If there are $M$ measurements available the optimal estimate will be found using all of them. Now if a subset $m<M$ is chosen, the estimate will be optimal for the reduced set and will have less certainty compared with the full optimization. Importantly, because bundle adjustment is being used, the error estimate will be a conservative one, guaranteeing consistency.

The question now is whether certain measurements can be removed to our advantage?

Figure 2 shows the Bayes net for a small bundle adjustment problem. Each measurement is conditioned on a landmark and the camera pose observing that landmark. Two well-known conditional independences are apparent, viz:

$$
\begin{aligned}
P\left(\mathbf{K}^{1}, \mathbf{K}^{2}, \mathbf{K}^{3} \mid \mathbf{X}^{1,2}\right) & =P\left(\mathbf{K}^{1} \mid \mathbf{X}^{1,2}\right) P\left(\mathbf{K}^{2} \mid \mathbf{X}^{1,2}\right) P\left(\mathbf{K}^{3} \mid \mathbf{X}^{1,2}\right) \\
P\left(\mathbf{X}^{1}, \mathbf{X}^{2} \mid \mathbf{K}^{1,2,3}\right) & =P\left(\mathbf{X}^{1} \mid \mathbf{K}^{1,2,3}\right) P\left(\mathbf{X}^{2} \mid \mathbf{K}^{1,2,3}\right) .
\end{aligned}
$$

The first allows the camera pose to be calculated if the landmarks are known (used in the tracking phase of PTAM, for example), and the second indicates that landmark estimations are independent given the camera trajectory (the basis of FastSLAM, for example). This is the case whether or not measurement $\mathbf{Z}_{13}$ is present. 
However, removing measurement $\mathbf{Z}_{13}$ exposes another conditional independence which we exploit in this work. Following its removal, keyframe 2 does not observe any of the landmarks in set 2 and keyframe 3 does not observe any of the landmarks in set 1 . This makes the keyframes and landmarks right of keyframe 1 conditionally independent of those to the left, as in

$$
P\left(\mathbf{X}^{1}, \mathbf{K}^{2}, \mathbf{X}^{2}, \mathbf{K}^{3} \mid \mathbf{K}^{1}\right)=P\left(\mathbf{X}^{1}, \mathbf{K}^{2} \mid \mathbf{K}^{1}\right) P\left(\mathbf{X}^{2}, \mathbf{K}^{3} \mid \mathbf{K}^{1}\right) .
$$

Figure 3 shows the Jacobian for the full system with the non zero blocks of the row that corresponds to measurement $\mathbf{Z}_{13}$ coloured in red (dark). Keyframe 1 has been chosen as the root keyframe from which all keyframes and landmarks derive their references ${ }^{2}$. In the top right of Figure 3 is the information matrix for the full system with the blocks created from just measurement $\mathbf{Z}_{13}$ again coloured red. The removal of measurement $\mathbf{Z}_{13}$ leads to the information matrix shown in the bottom right corner in which the two independent estimation problems can be seen clearly.

This independence can be used to partition the problem into two, one part concerned with estimating the local environment (Both the landmarks and keyframes) in the vicinity of the camera, and the second with estimating the remainder of the environment. The requirements of these two independent estimation problems differ. In particular, in the vicinity of the camera new keyframes need to be added and optimized quickly to keep pace with the movement of the camera and the fixed inter-frame time.

Importantly, the proposed method can respond to these requirements, as follows:

(1) The partition can be placed at any keyframe. The number of landmarks and keyframes following the partition can thus be chosen to allow exploration to continue in constant time. One can ensure strictly real-time performance.

(2) If the global optimization of the remaining landmarks and keyframes changes the pose of the selected partition keyframe, the landmarks and keyframes from the recent exploration are automatically updated to the new and better estimate, simply because their description is relative.

The only downside of the relative frame representation is that the density of the Jacobians of the image deviations with respect to the keyframes are denser than in standard bundle adjustment. This adds an extra overhead to the computation, but it does not increase the complexity.

\section{RELATIVE FRAME BUNDLE ADJUSTMENT APPLIED TO PARALLEL TRACKING AND MAPPING}

The Parallel Tracking and Mapping (PTAM) algorithm is suitable vehicle in which to apply and exploit relative frame bundle adjustment. As described in [10], the tracking process is a computationally inexpensive process that assumes the map is fixed, and uses features from each incoming frame to recover camera pose change. The mapping phase runs separately, computing structure and camera pose in keyframes, and is our principal concern here. Figure 4 shows an example of the typical feature and map densities recoverable.

\footnotetext{
${ }^{2}$ Only the local adjustment keyframes need be referenced to $\mathbf{K}^{1}$.
}

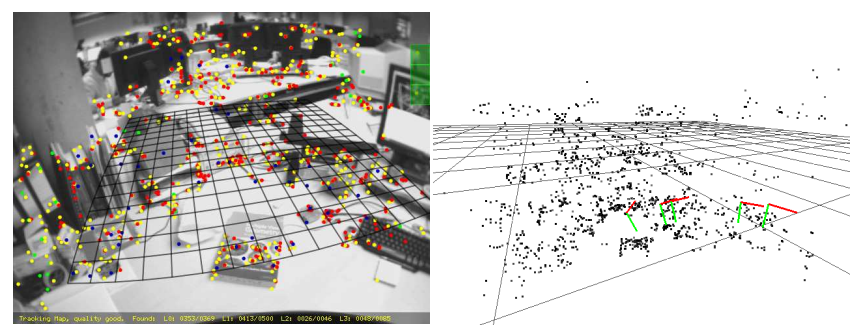

Fig. 4. A typical scene mapped by PTAM and the resulting map created.

\section{A. Tracking}

With a 3D map already initialized by the mapping phase, tracking proceeds by subsampling each newly captured greylevel image into a pyramid of four levels spanning three octaves, and computing FAST-10 [19] corner features at each scale. A prior of the camera pose is estimated from the previous frame using a constant (but decaying) velocity model and some fifty map points are projected into the image at the coarsest scale. Those that are matched to the current feature list (using location and appearance templates) are used to determine the camera pose change from two views using robust methods. In further iterations, more points are pulled into the optimization as the scale moves from coarse to fine.

The tracker is not affected by the replacement of standard bundle adjustment with the relative frame version and continues to use all the landmarks. For tracking it is efficient to calculate global positions of the landmarks after each bundle adjustment in the mapping phase. As the tracker runs at every frame, not just at keyframes, this avoids repeated computation of strings of Euclidean transformations to transform each landmark from its reference keyframe into the prior camera frame.

\section{B. Mapping}

To initialize a map at the outset, a camera viewpoint is selected by the user and its image and feature list becomes the zeroth keyframe $\mathbf{K}^{0}$. The camera is then translated to a new pose with sufficient care to allow features to be tracked using the image alone. This image is captured as the first keyframe $\mathbf{K}^{1}$. A robust version of Nistér's relative pose algorithm [20] is used to compute the change in pose and to triangulate map points. After further camera movement, a particular camera pose (along with relevant the image and 2D feature list) is nominated as a keyframe $\mathbf{K}^{k}$ whenever (i) tracking is deemed good; (ii) the camera has translated by a minimum distance from any previous keyframe; and (iii), to supress the volume of processing, around twenty frames have passed since the previous keyframe. To propose new landmarks $\mathbf{X}$, features are sought in the image which are unmatched, salient, and distant in the image from other matched features. To initialize a landmark, a search is made in the spatially closest keyframe for a correspondence, and the 3D position triangulated.

\section{Selective feature removal and the constant time bundle adjustment}

At this stage in standard PTAM a bundle adjustment of all keyframes and landmarks would take place. Instead, here 

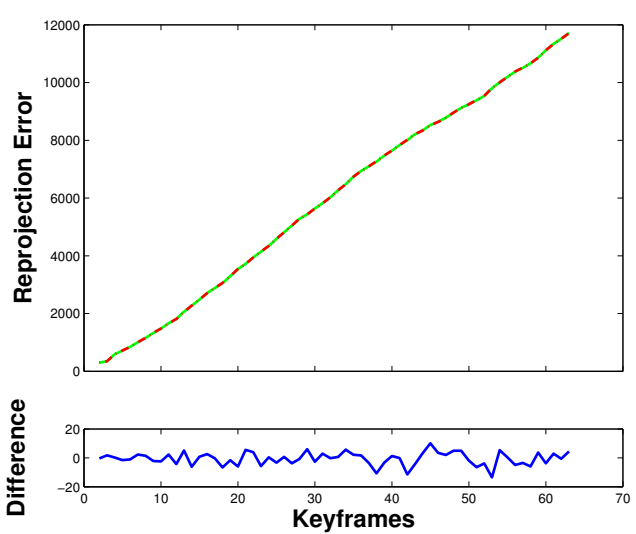

Fig. 5. Top, the reprojection errors for global (green,light) and relative (red,dark) coordinate frame bundle adjustments are barely different. As the number of keyframes, and hence measurements, increases the reprojection error for both relative and global coordinate frame bundle adjustment increases. The sight differences are shown below and arise from calculating the necessary transformations for reprojection using a series of transformations.

the selective (and temporary) removal of measurements in the local keyframes takes place, guaranteeing the conditional independence that in turn ensures that the bundle adjustments either side of the partition keyframe will be consistent. Note that removing measurements from keyframes earlier than the most recent never requires the removal of an established landmark. However, a landmark that has just been initialised will have to be removed if either of the two measurements that contributed to its initialisation is removed. It should be stressed again that all removals are temporary and apply only while the measurements relate to keyframes on the local side of the partition.

Now the the 3D positions $\left\{\mathbf{X}^{i}\right\}$, of the landmark points and the fixed number of keyframe camera poses $\left\{\mathbf{K}^{k}\right\}$ to the right (recent) side of the partition are optimized by relative frame bundle adjustment, allowing exploratory map-building in constant time.

\section{The more global bundle adjustment}

The last stage of the modified method is the bundle adjustment to the left of the partition. This is run as a separate non time-critical process. It uses the consistent estimates of existing keyframe camera poses and landmark positions as starting values, and pulls in any measurements belonging to the keyframes to the left of the partition that were excluded in the constant time adjustment. Although this adjustment could well be performed using the relative representation, it is more efficient in the global form for exactly the same reasons that tracking was more efficient — i.e. it avoids repeated multiplication of Euclidean transformations.

\section{Results}

The relative frame representation for bundle adjustment has been coded in $\mathrm{C}++$ and integrated into the PTAM framework presented as described. The experiments presented here compare the estimates produced by the global and relative frameworks by running the system on a recorded outdoor video sequence and obtaining the global solution using both representations. Both relative and global frame representations use the same initialization for the keyframe

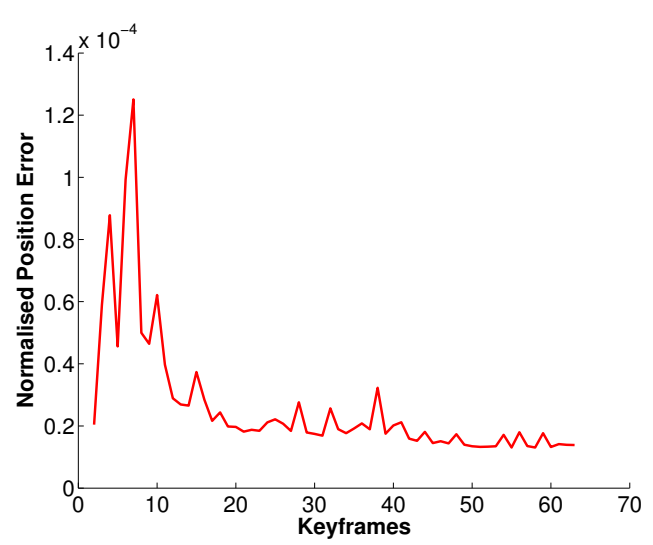

Fig. 6. The normalized Euclidean distance between the locations of the keyframes estimated by bundle adjustment in the global and relative representations.

poses and landmark locations. The same measurements are also used for both representations.

Figure 5 shows the reprojection error for both global and relative coordinate frames as more keyframes are added in a sequence. For both representations the bundle adjustment for all the keyframes is given enough time to converge fully. As expected, the total measurement error, measured in pixels, grows linearly with the number of keyframes for both relative and global representations. It is evident that both representations converge to the same measurement error with any difference due to machine precision.

The total Euclidean distance, normalized by the number of keyframes, between the camera pose estimated by relative and global frame bundle adjustment is shown in Figure 6. The normalized difference has been plotted to show that, as the size of the estimation increases, the difference in the estimates for each keyframe remains constant. The normalized $L_{2}$-norm between the camera orientations is very similar and not shown here.

The final result shown in Figure 7 is the time needed to complete relative frame bundle adjustment when exploring and mapping a new area. The estimates for landmarks and keyframes are sub-optimal as measurements have been excluded to ensure conditional independence, but they are conservative estimates for the true solution, as discussed in Section II-A. In this experiment the number of keyframes involved in each estimation is capped at a maximum of thirty. (A method for adaptively choosing this number awaits development of fully optimized code.) The average time over five runs taken to compute the solution is seen to rise initially as the number of keyframes increases from two to the cap at thirty. Thereafter the time taken for each estimation then fluctuates with the number of landmarks involved and the density of the Jacobians with respect to the keyframes, but is evidently $O(1)$.

\section{Conclusions}

This paper has presented the combination of the relative frame representation of bundle adjustment [17] with the parallel tracking and mapping method [10] to provide many of the key characteristics of a real-time system for the recovery of structure from motion. 


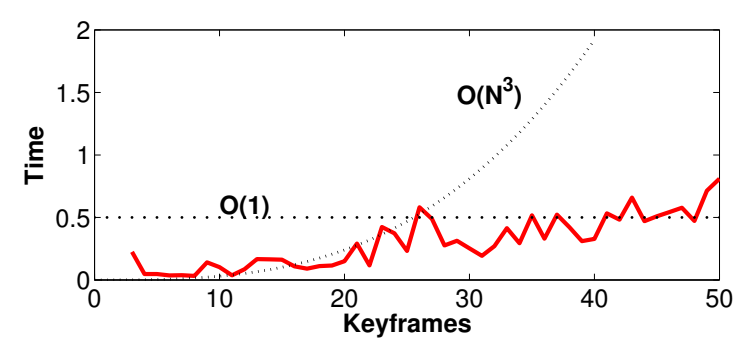

Fig. 7. The time (in seconds) taken to complete relative bundle adjustment when new mapping is occuring. In this example the number of keyframes which are adjusted is limited to thirty and the times taken have been averaged over five runs. At the start of the experiment the time taken to find the solution increases as the number of keyframes grows from two to thirty and then levels off as expected with a problem of constant size. Note that the number of keyframes on the abcissa is the number of keyframes collected so far and not the number of keyframes being estimated.

The selective and temporary neglect of certain measurements introduces a conditional independence which combined with the relative frame representation of bundle adjustment allows the solution to be conveniently broken into two parts that nonetheless deliver globally consistent results. The first runs in constant time on a fixed number of the most immediately local landmarks and camera keyframe poses, and allows fixed-time exploration of the scene. The other part performs a more global optimization on the remaining landmarks and keyframes using all available measurements, and runs as a separate batch process. Importantly, this allows exploration and mapping of new areas to be pursued concurrently with the optimal solution computation. At any time a conservative estimate of the whole solution can be found simply by appending the local solution to the more global solution. Furthermore, the relative frame representation allows improvements made by the global adjustment to immediately be transferred to the local adjustment. Experiments show that the relative frame representation for bundle adjustment produces the same results as the global frame representation up to machine error.

\section{A. Future Work}

There are two strands to future work. The first is to make more thorough comparisons between relative and global frame bundle adjustment, to include an analysis of convergence behaviours in terms of numbers of iterations and regions of convergence. The computational penalty imposed by denser Jacobians in the relative frame representation needs characterising: this could not be done fairly here because the relative frame implementation has not been optimized to the same degree as unmodifield PTAM.

The second strand involves a practical exploration of the performance of the relative frame bundle adjustment and the partitioning approach to exploration in over large areas. Key to doing this is the implementation of the exploratory adjustment and global adjustment as separate processes, perhaps on separate processors. This relative frame representation make inter-process communication of updated results particularly straightforward. The partitioning will be particularly useful when closing large loops. The optimization of the keyframes around the loop could be carried out while the exploration re-maps the earlier section. This would give more time to calculate the optimal solution for the loop close. The full map optimization does not need to be computed in constant time as long as it is calculated independently of the map building occuring in the exploration thread.

\section{REFERENCES}

[1] M. Montemerlo, S. Thrun, D. Koller, and B. Wegbreit, "FastSLAM: A factored solution to the simultaneous localization and mapping problem," in Proceedings of the AAAI National Conference on Artificial Intelligence, Edmonton, Canada, 2002.

[2] T. Bailey, J. Nieto, and E. Nebot, "Consistency of the fastslam algorithm," in Proc Int Conf on Robotics and Automation, Orlando FL, USA, May, 2006, pp. 424-429.

[3] R. Smith and P. Cheeseman, "On the representation and estimation of spatial uncertainty," Int. Journal of Robotics Research, vol. 5, no. 4, pp. 56-68, 1986.

[4] J. Leonard and H. Durrant-Whyte, Directed Sonar Sensing for Mobile Robot Navigation. Boston MA: Kluwer Academic, 1992.

[5] D. Chekhlov, M. Pupilli, W. Mayol-Cuevas, and A. Calway, "Realtime and robust monocular SLAM using predictive multi-resolution descriptors," in Proc 2nd Int Symp on Visual Computing, Lake Tahoe Nevada, November 6-8, 2006, pp. 276-285.

[6] T. Bailey, J. Nieto, J. Guivant, and M. S. an E. Nebot, "Consistency of the EKF-SLAM algorithm," in Proc IEEE/RSJ Conf on Intelligent Robots and Systems, Beijing, China, October 9-15, 2006, pp. 35623568.

[7] S. Julier and J. Uhlmann, "A counter example to the theory of simultaneous localization and map building," in Proc Int Conf on Robotics and Automation, Seoul, Korea, May 21-26, 2001, pp. 42384243.

[8] S. Holmes, G. Klein, and D. W. Murray, "A Square Root Unscented Kalman Filter for visual monoSLAM," in Proc Int Conf on Robotics and Automation, Pasadena CA, USA, May 19-23, 2008.

[9] C. McGlone, E. Mikhail, and J.Bethel, Manual of Photogrammetry, 5th Edition. Bethesda, MD: American Society of Photogrammetry and Remote Sensing, 2004.

[10] G. Klein and D. Murray, "Parallel tracking and mapping for small AR workspaces," in Proc. Sixth IEEE and ACM International Symposium on Mixed and Augmented Reality, Nara, Japan, November 2007.

[11] R. M. Eustice, H. Singh, and J. J. Leonard, "Exactly Sparse DelayedState Filters for View-Based SLAM," IEEE Transactions on Robotics, vol. 22, no. 6, pp. 1100-1114, December 2006.

[12] M. Kaess, A. Ranganathan, and F. Dellaert, "iSAM: Fast Incremental Smoothing and Mapping with Efficient Data Association," in Proc Int Conf on Robotics and Automation, Rome, Italy, April 10-14, 2007.

[13] M. Bosse, P. Newman, J. Leonard, and S. Teller, "Simultaneous localization and map building in large-scale cyclic environments using the atlas framework," Int. Journal of Robotics Research, vol. 23, no. 12, pp. 1113-1139, 2004.

[14] E. Eade and T. Drummond, "Unified loop closing and recovery for real time monocular slam," in Proc 19th British Machine Vision Conference, Leeds, Sept 2008.

[15] A. Davison, I. Reid, N. Molton, and O. Stasse, "MonoSLAM: Realtime single camera SLAM," IEEE Transactions on Pattern Analysis and Machine Intelligence, vol. 29, no. 6, pp. 1052-1067, 2007.

[16] P. Piniés and J. D. Tardós, "Scalable slam building conditionally independent local maps," in Proc IEEE/RSJ Conf on Intelligent Robots and Systems, San Diego CA, USA, October 29-November 2, 2007, pp. 3466-3471.

[17] G.Sibley, "Relative bundle adjustment," Department of Engineering Science, Oxford University, Tech. Rep. 2307/09, January 2009.

[18] W. Triggs, P. McLauchlan, R. Hartley, and A. Fitzgibbon, "Bundle adjustment: A modern synthesis," in Vision algorithms: theory and practice, LNCS, W. Triggs, A. Zisserman, and R. Szeliski, Eds. Springer Verlag, 2000.

[19] E. Rosten and T. Drummond, "Machine learning for high-speed corner detection," in European Conference on Computer Vision, vol. 1, May 2006, pp. 430-443.

[20] D. Nistér, "An efficient solution to the five-point relative pose problem," IEEE Transactions on Pattern Analysis and Machine Intelligence, vol. 26, no. 6, pp. 756-770, June 2004. 